\title{
On the Sum of Reciprocals of Mersenne Primes
}

\author{
Yoshihiro Tanaka \\ Faculty of Economics and Business, Hokkaido University, Sapporo, Japan \\ Email: tanaka@econ.hokudai.ac.jp
}

How to cite this paper: Tanaka, Y. (2017) On the Sum of Reciprocals of Mersenne Primes. American Journal of Computational Mathematics, 7, 145-148.

https://doi.org/10.4236/ajcm.2017.72012

Received: March 31, 2017

Accepted: June 5, 2017

Published: June 8, 2017

Copyright $\odot 2017$ by author and Scientific Research Publishing Inc. This work is licensed under the Creative Commons Attribution International License (CC BY 4.0).

http://creativecommons.org/licenses/by/4.0/

\begin{abstract}
The sum of reciprocals of Mersenne primes converges to $0.51645417894078856533 \cdots$, which is an example of a probably infinite subset of primes whose sum of reciprocals is finite and can be computed accurately. This value is larger than $\sum_{\{q \in \mathbb{P} \mid p \geq 3\}} \frac{1}{q-1}$, where $\mathbb{P}$ is the set of perfect powers of prime numbers.
\end{abstract}

\section{Keywords}

Mersenne Primes, the Sum of Reciprocals, Unresolved Problem

\section{Introduction}

Since monk Marine Mersenne studied the primality of $M_{n}=2^{n}-1$ in 1644, Mersenne primes, i.e., $M_{n}=2^{n}-1 \quad\left(M_{n}\right.$ : prime), have been developed by numerous researchers, such as Euler, Lucas, Pervouchine, Cole, and Powers, and in recent years, by GIMPS (Great Internet Mersenne Prime Search).

If $M_{n}$ is prime, then $n$ is also prime, because if $n=a b,(a, b \geq 2)$, then $M_{n}=11 \cdots 1$ ( $a b$ digits in binary) can be divided by $1 \cdots 1$ ( $a$ digits in binary). However, the converse is not true, for example, $M_{11}=2047=23 \times 89$.

In addition, it is well known that all even perfect numbers (odd perfect numbers are unknown) are generated by $2^{n-1} M_{n}$, if and only if $M_{n}$ is prime.

The current Mersenne prime numbers are denoted by $M_{n}$, for $n=2,3,5,7,13,17,19,31,61,89,107,127,521, \cdots$. The most recent Mersenne prime number is $M_{74207281}$ (22338618 digits), which was developed in January 2016.

\section{Bounds for the Sum of Reciprocals of Mersenne Primes}

We begin by defining the notation. We define

$$
S \equiv\{2,3,5,7,13,17,19,31,61,89,107,127,521, \cdots\}
$$

$i_{k}: k$-th number in $S$. 
Theorem 1.

$$
\sum_{k=1}^{N} \frac{1}{M_{i k}}<\sum_{M_{n}: \text { primes }} \frac{1}{M_{n}}<\sum_{k=1}^{N} \frac{1}{M_{i k}}+2^{2-i_{N+1}}, \forall i_{N} \in S
$$

Proof.

$$
\begin{aligned}
\sum_{M_{n}: \text { primes }} \frac{1}{M_{n}} & =\sum_{i \in S} \frac{1}{M_{i}}=\sum_{k=1}^{N} \frac{1}{M_{i k}}+\sum_{k=N+1}^{\infty} \frac{1}{M_{i k}}<\sum_{k=1}^{N} \frac{1}{M_{i k}}+\sum_{k=N+1}^{\infty} \frac{1}{2^{i_{k}}-1} \\
& <\sum_{k=1}^{N} \frac{1}{M_{i k}}+\sum_{k=N+1}^{\infty} \frac{1}{2^{i_{k}-1}}=\sum_{k=1}^{N} \frac{1}{M_{i k}}+2^{2-i_{N+1}}
\end{aligned}
$$

We can effectively calculate $\sum_{M_{n}: \text { primes }} \frac{1}{M_{n}}$, as $2^{2-i_{N+1}}$ rapidly converges to 0 . For example, if we consider $N=8$, we obtain

$$
0.51645417894078856489 \cdots<\sum_{M_{n} \text { :primes }} \frac{1}{M_{n}}<0.51645417894078856663 \cdots,
$$

which provides the value of $\sum_{M_{n} \text { :primes }} \frac{1}{M_{n}}$ up to 17 decimal digits. If we consider $N=12$, we can precisely calculate the sum of reciprocals of Mersenne primes up to 156 decimal digits, which is given by

0.516454178940788565330487342971522858815968553415419701441931

065273568701440212723499154883293666215374032432110836569575

$419140470924868317486037285294641634 \ldots$

\section{Comments}

According to the Goldbach-Euler theorem [1],

$$
\sum_{q \in \mathbb{P}} \frac{1}{q-1}=\frac{1}{3}+\frac{1}{7}+\frac{1}{8}+\frac{1}{15}+\frac{1}{24}+\cdots=1,
$$

where $\mathbb{P} \equiv\left\{p^{i} \mid p\right.$ is a prime number $\geq 2$ and $\left.i \geq 2\right\}$ is the set of perfect powers of prime numbers.

Theorem 2. The sum of reciprocals of Mersenne prime numbers is larger than that of $q-1$ where $q \in \mathbb{P} \mid p \geq 3$, namely,

$$
\sum_{M_{n}: \text { primes }} \frac{1}{M_{n}}>\sum_{\{q \in \mathbb{P} \mid p \geq 3\}} \frac{1}{q-1}
$$

Proof. It holds that

$$
\frac{1}{M_{2}}+\frac{1}{M_{3}}+\frac{1}{M_{5}} \approx 0.50844854 \cdots<\sum_{M_{n} \text { :primes }} \frac{1}{M_{n}} .
$$

Considering that $M_{n}$ : primes $\in 2^{i}-1(i \geq 2)$, it follows from Goldbach-Euler theorem that

$$
\sum_{M_{n}: \text { primes }} \frac{1}{M_{n}}+\sum_{\{q \in \mathbb{P} \mid} \frac{1}{p \geq 3\}} \frac{1}{q-1}<\sum_{i \geq 3} \frac{1}{2^{i}-1}+\sum_{\{q \in \mathbb{P} \mid} \frac{1}{p \geq 3\}} \frac{1}{q-1}=1 .
$$

Hence, 


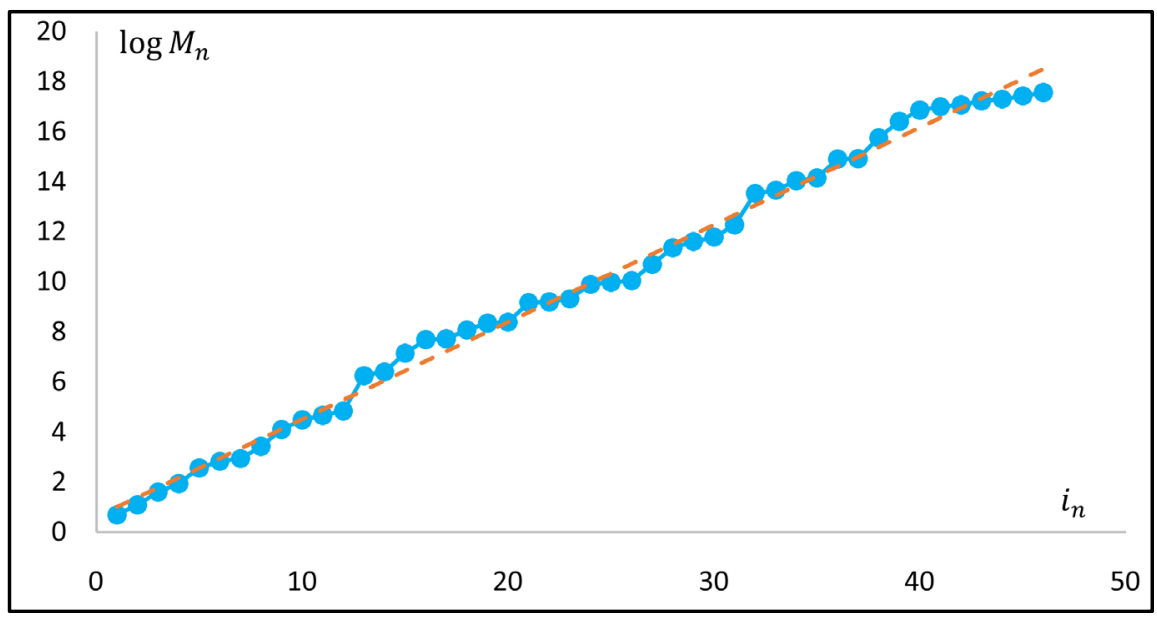

Figure 1. Relationship between $i_{n}$ and $\log M_{n}$.

$$
\sum_{\{q \in \mathbb{P} \mid p \geq 3\}} \frac{1}{q-1}<\frac{1}{2}<\sum_{M_{n}: \text { primes }} \frac{1}{M_{n}}
$$

since $\frac{1}{2}<\sum_{M_{n}: \text { primes }} \frac{1}{M_{n}}$.

We should note that the sum of reciprocals of prime numbers appears to converge numerically; however, it is infinite, which is proved in, e.g., Hardy and Wright [2]. Therefore, Mersenne primes are considerably sparse subsequences of prime numbers.

In the case of twin primes, the value of the sum of reciprocals of twin primes is shown to be bounded above by Brun [3] and is estimated as

$$
\left(\frac{1}{3}+\frac{1}{5}\right)+\left(\frac{1}{5}+\frac{1}{7}\right)+\left(\frac{1}{11}+\frac{1}{13}\right)+\left(\frac{1}{17}+\frac{1}{19}\right)+\cdots \approx 1.9021605823 \pm 8 \times 10^{-10},
$$

which is known as Brun's constant (however, it is an estimation). Even though the problem that whether twin primes are infinite is still unsolved, Zhang [4] presented an important result, which states that a constant $<7 \times 10^{7}$ exists between two successive primes that are infinite. If we can lower the upper bound by 4 , the twin prime conjecture will be solved. The Polymath8 project has reduced the upper bound by four digits.

In addition, the problem that whether Mersenne primes are infinite is still unresolved. Figure 1 shows the relationship between $i_{n}$ and $\log M_{n}$.

Denote $m(x)$ the number of Mersenne primes that do not exceed $x$. Then, as $m(x)=i_{n}$ for $x=M_{n}$, it seems from Figure 1 that

$$
m(x) \propto \log x+c,
$$

numerically, where $c$ is a constant. In other words, the number of Mersenne primes tends to increase by a constant per digit.

\section{References}

[1] Bibiloni, L., Viader, P. and Paradís, J. (2006) On a Series of Goldbach and Euler. The American Mathematical Monthly, 113, 206-220. 
https://doi.org/10.2307/27641889

[2] Hardy, G.H. and Wright, E.H. (1979) An Introduction to the Theory of Number. 5th Edition, Oxford University Press, New York.

[3] Brun, V. (1919) La série

$\frac{1}{5}+\frac{1}{7}+\frac{1}{11}+\frac{1}{13}+\frac{1}{17}+\frac{1}{19}+\frac{1}{29}+\frac{1}{31}+\frac{1}{41}+\frac{1}{43}+\frac{1}{59}+\frac{1}{61}+\cdots \quad$ les dénominateurs sont nombres premiers jumeaux est convergente où finie. Bulletin des Sciences Mathématiques, 43, 124-128. (In French)

[4] Zhang, Y. (2014) Bounded Gaps between Primes. Annals of Mathematics, 179, 1121-1174. https://doi.org/10.4007/annals.2014.179.3.7

Submit or recommend next manuscript to SCIRP and we will provide best service for you:

Accepting pre-submission inquiries through Email, Facebook, LinkedIn, Twitter, etc. A wide selection of journals (inclusive of 9 subjects, more than 200 journals)

Providing 24-hour high-quality service

User-friendly online submission system

Fair and swift peer-review system

Efficient typesetting and proofreading procedure

Display of the result of downloads and visits, as well as the number of cited articles Maximum dissemination of your research work

Submit your manuscript at: http://papersubmission.scirp.org/

Or contact ajcm@scirp.org 